\title{
Filter Bank Frame Expansions with Erasures
}

\author{
Jelena Kovačević, \\ Fellow, IEEE \\ Pier Luigi Dragotti \\ Bell Labs \\ EPFL \\ Murray Hill, NJ \\ jelena@bell-labs.com \\ Lausanne, Switzerland \\ Pierluigi.Dragotti@epfl.ch
}

and

Vivek K Goyal

Member, IEEE

\author{
Digital Fountain \\ Fremont, CA \\ v.goyal@ieee.org
}

\begin{abstract}
We study frames for robust transmission over the Internet. In our previous work, we used quantized finitedimensional frames to achieve resilience to packet losses; here, we allow the input to be a sequence in $\ell_{2}(\mathbb{Z})$ and focus on a filter-bank implementation of the system. We present results in parallel, $\mathbb{R}^{N}$ or $\mathbb{C}^{N}$ versus $\ell_{2}(\mathbb{Z})$, and show that uniform tight frames, as well as newly introduced strongly uniform tight frames, provide the best performance.
\end{abstract}

Keywords-Frames, multiple descriptions, oversampled filter banks.

\section{INTRODUCTION}

Aaron D. Wyner, to whom this issue and this paper are dedicated, had a profound impact on information theory and on his colleagues - including the first and third authors. Amongst Wyner's varied contributions were the conception and development of source coding problems that generalized Shannon's basic point-to-point communication problem [1], [2], [3], [4], [5]. Network source coding problems inspired in part by Wyner's work currently occupy many theoreticians and compression practitioners.

This paper concerns the analysis of one framework for communicating infinite sequences over a set of parallel channels, each of which is either noiseless or does not work at all. The channel model gives a general form of multiple description coding [6]. The transmitted information is generated with a filter bank and scalar quantization, as shown in Fig. 1. The filter bank implements a frame expansion; thus, the structure itself and the techniques for analysis and design are generalizations of results for finite-dimensional vectors in [7], [8].

\section{A. Frames}

Frames have become ubiquitous. They started as a mathematical theory by Duffin and Schaeffer [9], who provided an abstract framework for the idea of time-frequency atomic decomposition by Gabor [10]. The theory then laid largely dormant until 1986 with the publication of the work by Daubechies, Grossman and Meyer [11]. Since then, frames have evolved into a state-of-the-art signal processing tool.

The mathematics of frames can be found in several excellent sources. The original work by Duffin and Schaeffer introduced frames for Hilbert spaces [9]. The paper by Daubechies, Grossman and Meyer [11] discusses applications to wavelet and Gabor transforms. A beautiful tutorial on the art of frame theory was written by
Casazza [12]. Some particular classes of frames have been extensively studied: Gabor frames (also called WeylHeisenberg frames) are described by Heil and Walnut in [13] and by Casazza in [14], while the paper [15] and the book [16] by Daubechies offer excellent introductions to frames and in particular, wavelet and Gabor frames.

Frames, or redundant representations, have been used in different areas under different guises. Perfect reconstruction oversampled filter banks are equivalent to frames in $\ell_{2}(\mathbb{Z})$. The authors in [17], [18], [19] describe and analyze such frames. Frames show resilience to additive noise as well as numerical stability of reconstruction [16]. They have also demonstrated resilience to quantization [20], [21]. Several works exploit the greater freedom to capture significant signal characteristics which frames provide [22], [23], [24]. Frames have been used to design unitary space-time constellations for multiple-antenna wireless systems [25]. Finally, although a well-known result by a Russian mathematician M.A. Naimark ${ }^{1}$-Naimark's Theorem [26]-has been widely used in frame theory in the past few years [8], [27], only recently have researchers recast certain quantum measurement results in terms of frames [28], [29].

The bibliography on frames is vast; the list given above is just a sample. The reader is encouraged to check the references above and the ones within for more uses of frames and further technical details.

\section{B. Structure of the Proposed System}

As in previous work of two of the authors [8], our aim is to exploit the resilience of frame expansions to coefficient losses. This resilience is a result of the redundancy a frame representation brings. In the earlier work, the frame elements belong to $\mathbb{R}^{N}$ (or $\mathbb{C}^{N}$ ) and can be seen as filters in a block-transform filter bank. Here, we investigate frames with elements in $\ell_{2}(\mathbb{Z})$; they can be seen as filters in a general, oversampled filter bank.

Consider the model depicted in Fig. 1. An input sequence $x[n]$ is fed through an $M$-channel finite-impulse response (FIR) filter bank followed by downsampling by $N$ $(N<M)$. The $M$ output sequences are then separately scalar quantized with uniform scalar quantizers and sent over $M$ different channels. Each channel either works perfectly or not at all. The decoder receives only $M-e$ of the quantized output sequences, where $e$ is the number of erasures during the transmission. We assume there are no

\footnotetext{
${ }^{1}$ A common alternative spelling of Naimark is Neumark.
} 


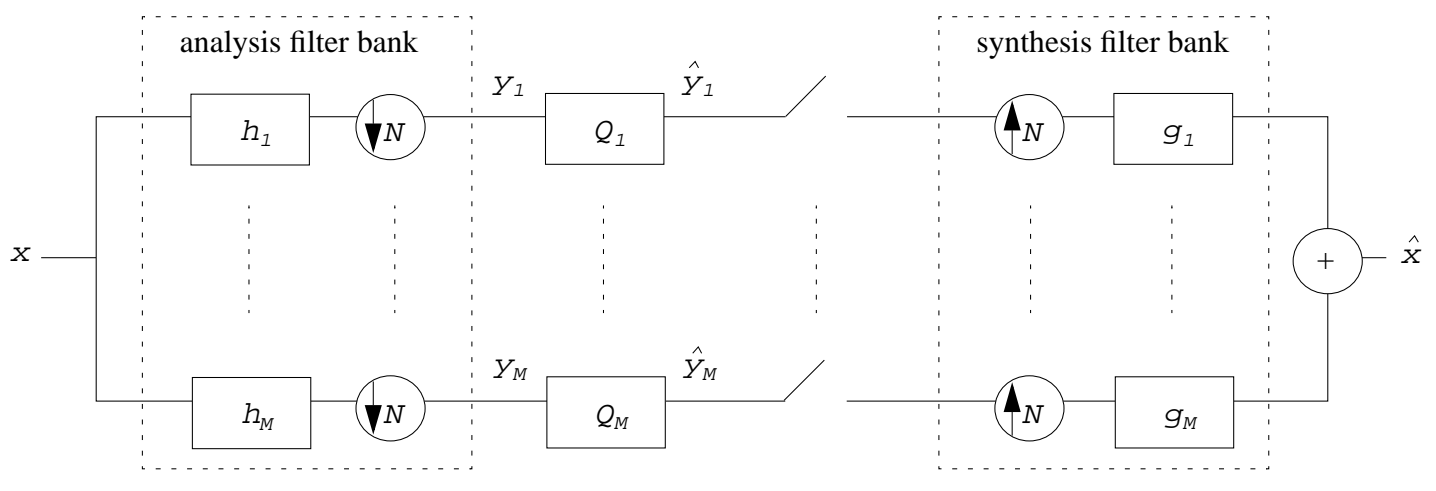

Fig. 1. Abstraction of a lossy network with a frame expansion implemented by an oversampled FIR filter bank. An input sequence $x[n]$ is fed through an $M$-channel finite-impulse response (FIR) filter bank that includes downsampling by $N(N<M)$. The $M$ output sequences are then separately scalar quantized with uniform scalar quantizers and sent over $M$ different channels. Each channel either works perfectly or not at all. The decoder receives only $M-e$ of the quantized output sequences, where $e$ is the number of erasures during the transmission. We assume there are no more than $M-N$ erasures. The reconstruction process is performed by the synthesis filter bank. The choice of synthesis filters depends on which channels are received.

more than $M-N$ erasures. The reconstruction process is linear. We wish to find properties of the filter banks that minimize the mean square error (MSE) between the input and the reconstructed sequences.

To analyze cases with more than $M-N$ erasures requires a statistical model for the input sequence. In [30], [31], [32], the input sequence is a stationary Gaussian source; in [30], [32] the case $M=N=2$ and one erasure is considered, while in [31] the case $M=3$ and $N=2$ and up to two erasures is analyzed. In this work we do not make any assumptions on the input source. Rather, a statistical model for the quantization error makes the reconstruction quality depend only on the characteristics of the filter bank.

We first go through the basics of frame expansions in $\mathbb{H}_{N}$ (where $\mathbb{H}_{N}$ denotes a finite-dimensional space such as $\mathbb{R}^{N}$ or $\mathbb{C}^{N}$ ) and $\ell_{2}(\mathbb{Z})$. We introduce the notion of strongly uniform frames and discuss several examples. We then quantize the frame coefficients and find the MSE. Finally, we let some coefficients be erased (mimicking the losses in a network) and discuss the effect on both the structure of the frame and the MSE. Although we could present only the results for $H=\ell_{2}(\mathbb{Z})$ and specialize them to $H=\mathbb{H}_{N}$ when the filter length is $N$, we present the known results for $\mathbb{H}_{N}[8]$ and the new ones for $\ell_{2}(\mathbb{Z})$ in parallel; the simple geometry of $\mathbb{H}_{N}$ makes results feel more intuitive.

A few words about notation: superscript ${ }^{*}$ denotes the Hermitian transpose (complex conjugation as well as transposition in case of vectors and matrices). In the filter bank literature, it is customary to denote matrices by bold capital letters; we will depart from this convention here to be consistent with the frame notation.

\section{Simple Example}

We are given the following set of three vectors in $\mathbb{R}^{2}$ :

$$
\begin{aligned}
\Phi & =\left\{\varphi_{1}^{*}, \varphi_{2}^{*}, \varphi_{3}^{*}\right\} \\
& =\left\{(0,1)^{*},(-\sqrt{3} / 2,-1 / 2)^{*},(\sqrt{3} / 2,-1 / 2)^{*}\right\}
\end{aligned}
$$

(see Fig. 2). This set is obviously not a basis, since it has more vectors (three) than needed to represent vectors in $\mathbb{R}^{2}$ (two). However, it can still be used to represent vectors from $\mathbb{R}^{2}$ (albeit with linearly dependent vectors, $\left.\varphi_{1}=-\varphi_{2}-\varphi_{3}\right)$. We can write any $x \in \mathbb{R}^{2}$ as

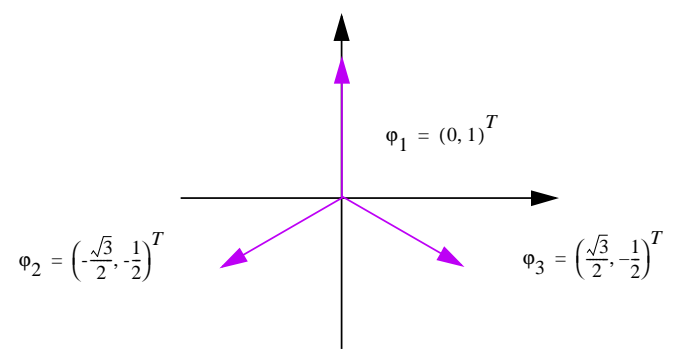

Fig. 2. Mercedes-Benz frame: A uniform tight frame with three vectors in two dimensions. It is a representative of the whole class of uniform tight frames with $N=2, M=3$.

$$
\begin{aligned}
x=\left(\begin{array}{l}
x_{1} \\
x_{2}
\end{array}\right)= & \frac{2}{3} x_{2}\left(\begin{array}{l}
0 \\
1
\end{array}\right)+\frac{2}{3}\left(-\frac{\sqrt{3}}{2} x_{1}-\frac{1}{2} x_{2}\right)\left(\begin{array}{c}
-\sqrt{3} / 2 \\
-1 / 2
\end{array}\right)+ \\
& +\frac{2}{3}\left(\frac{\sqrt{3}}{2} x_{1}-\frac{1}{2} x_{2}\right)\left(\begin{array}{c}
\sqrt{3} / 2 \\
-1 / 2
\end{array}\right) .
\end{aligned}
$$

This expression looks like it came out of the blue; however, if we look more closely, we note that it can be expressed as follows:

$$
x=\frac{2}{3} \sum_{i=1}^{3}\left\langle\varphi_{i}, x\right\rangle \varphi_{i} .
$$

The above equation looks suspiciously like the expansion formula using an orthonormal basis with basis vectors $\varphi_{i}$. That is, except for the term $2 / 3$. However, even that seems to make sense; we are normalizing our expansion by the factor that tell us what the "redundancy" of the system is, that is, how many more vectors we have than what we 
would have needed to represent vectors in $\mathbb{R}^{2}$. Moreover, the energy in the transform coefficients $X_{i}$ with

$$
X_{i}=\left\langle\varphi_{i}, x\right\rangle, \quad i=1,2,3, \quad X=\left(X_{1}, X_{2}, X_{3}\right)^{*},
$$

is

$$
\|X\|^{2}=\sum_{i=1}^{3}\left|\left\langle\varphi_{i}, x\right\rangle\right|^{2}=\frac{3}{2}\|x\|^{2}
$$

that is, it is $(3 / 2)$ times that in the input vector. Fairly intuitive. ${ }^{2}$

Thus, it seems that what we obtained is slightly more particular than just a random collection of three vectors. In fact, what we have is a frame; and not just any frame, it is a uniform tight frame (this particular frame is called a Mercedes-Benz (MB) frame). ${ }^{3}$ We can thus think of this frame as a generalization of an orthonormal basis. As we will see in Section III-C.3, a tight frame satisfies $F^{*} F=A I$ where $F$ is the matrix having elements of $\Phi$ as its rows. A uniform frame contains all vectors of norm 1 , as is the case in our example, and $A$ is the redundancy ratio $3 / 2$.

Suppose now that we perturb our frame coefficients by adding white noise $w_{i}$ to the channel $i$, where $E\left[w_{i}\right]=0$, $E\left[w_{i} w_{k}\right]=\sigma^{2} \delta_{i k}$ for $i, k=1,2,3$. Using (2), we can find the error of the reconstruction,

$$
\begin{aligned}
x-\hat{x} & =\frac{2}{3} \sum_{i=1}^{3}\left\langle x, \varphi_{i}\right\rangle \varphi_{i}-\frac{2}{3} \sum_{i=1}^{3}\left(\left\langle x, \varphi_{i}\right\rangle+w_{i}\right) \varphi_{i} \\
& =-\frac{2}{3} \sum_{i=1}^{3} w_{i} \varphi_{i} .
\end{aligned}
$$

Then the averaged mean-squared error per component is

$$
\begin{aligned}
\mathrm{MSE} & =\frac{1}{2} E\|x-\hat{x}\|^{2}=\frac{1}{2} E\left\|\frac{2}{3} \sum_{i=1}^{3} w_{i} \varphi_{i}\right\|^{2} \\
& =\frac{1}{2} \sigma^{2} \frac{4}{9} \sum_{i=1}^{3}\left\|\varphi_{i}\right\|^{2}=\frac{2}{9} \sigma^{2} \cdot 3=\frac{2}{3} \sigma^{2},
\end{aligned}
$$

since all the frame vectors have norm 1 .

The above result will show another particular property of the frame we chose so "randomly". Namely, among all other frames with three norm- 1 frame vectors in $\mathbb{R}^{2}$, this particular one (and the others in the same class, as will be shown later) minimizes the MSE. We can see this if we perturb the first vector by $\alpha$ radians clockwise. ${ }^{4}$ It then becomes $\varphi_{1}=(\sin \alpha, \cos \alpha)^{*}$. Denote the new frame matrix by $F_{1}$ and find the new left inverse of $F_{1}$. Repeating the above calculation, we get that the MSE is

$$
\mathrm{MSE}=\frac{1}{2} \sigma^{2} \frac{3}{9 / 4-(\sin \alpha)^{2}} .
$$

\footnotetext{
${ }^{2}$ We will see later that this is true only for the so-called uniform tight frames.

${ }^{3}$ We call this frame a Mercedes-Benz frame since the geometric configuration of its vectors brings to mind the Mercedes-Benz car logo.

${ }^{4} \mathrm{Of}$ course, perturbing just the first vector does not give us the most general frame in $\mathbb{R}^{2}$; we neglect this issue for simplicity and refer the reader to [8] for a more general treatment.
}

This MSE is minimized when $\alpha=0$; we are back to the MB frame! Moreover, our discussion justifies the statement in the introduction that frames provide resilience to quantization; with an orthonormal basis, ${ }^{5}$ the MSE $=\sigma^{2}$ (take the usual orthonormal basis $\varphi_{1}=(1,0)^{*}, \varphi_{2}=(0,1)^{*}$ and repeat the above MSE calculations), while with our frame, the $\mathrm{MSE}=(2 / 3) \sigma^{2}$, a reduction of the error by one third.

Recall, however, that our intention was to use frames to provide robustness to losses. Assume, thus, that one of the quantized coefficients is lost, for example, $X_{2}$. Does our MB frame have further nice properties when it comes to losses? Note first, that even with $\varphi_{2}$ not present, we can still use $\varphi_{1}$ and $\varphi_{3}$ to represent any vector in $\mathbb{R}^{2}$. The expansion formula is just not as elegant:

$$
x=\sum_{i=1,3}\left\langle\varphi_{i}, x\right\rangle \tilde{\varphi}_{i},
$$

with

$$
\tilde{\varphi}_{1}=\left(\begin{array}{c}
1 / \sqrt{3} \\
1
\end{array}\right), \quad \tilde{\varphi}_{3}=\left(\begin{array}{c}
2 / \sqrt{3} \\
0
\end{array}\right) .
$$

Repeating the same calculations as above for the MSE, we get that

$$
\begin{aligned}
\operatorname{MSE}_{\{2\}} & =\frac{1}{2} E\|x-\hat{x}\|^{2}=\frac{1}{2} E\left\|\sum_{i=1,3} w_{i} \tilde{\varphi}_{i}\right\|^{2} \\
& =\frac{1}{2} \sigma^{2} \sum_{i=1,3}\left\|\tilde{\varphi}_{i}\right\|^{2}=\frac{4}{3} \sigma^{2},
\end{aligned}
$$

that is, twice the MSE without erasures. However, the above calculations do not tell us anything about whether there is another frame with a lower MSE. In fact, given that one element is erased, does it really matter what the original frame was?

It turns out that it does. In fact, among all frames with three norm- 1 frame vectors in $\mathbb{R}^{2}$, the MSE averaged over all possible erasures of one coefficient is minimized when the original frame is tight [8]. For a hint of the general result, as before, perturb the first vector by $\alpha$ (and as before, be aware that this does not give us the most general uniform frame). Erasing one element at a time, compute the new inverse of the matrix formed by the remaining vectors and compute the MSE in each case. We get

$$
\begin{aligned}
\operatorname{MSE}_{\{1\}} & =\frac{4}{3} \sigma^{2}, \\
\mathrm{MSE}_{\{2\}} & =\sigma^{2} \frac{4}{2+\cos 2 \alpha+\sqrt{3} \sin 2 \alpha}, \\
\operatorname{MSE}_{\{3\}} & =\sigma^{2} \frac{4}{2+\cos 2 \alpha-\sqrt{3} \sin 2 \alpha} .
\end{aligned}
$$

The average MSE with one erasure is then

$$
\begin{aligned}
\mathrm{MSE}_{1} & =\frac{1}{3}\left(\mathrm{MSE}_{\{1\}}+\mathrm{MSE}_{\{2\}}+\mathrm{MSE}_{\{3\}}\right) \\
& =\frac{4}{9} \sigma^{2} \cdot \frac{15+10 \cos 2 \alpha+2 \cos 4 \alpha}{(1+2 \cos 2 \alpha)^{2}}
\end{aligned}
$$

\footnotetext{
${ }^{5}$ The orthonormal basis minimizes the MSE among all twodimensional bases; take, for example, $\varphi_{1}=(\cos \alpha, \sin \alpha)^{*}, \varphi_{2}=$ $(0,1)^{*}$. The MSE is $\left(2 \sigma^{2}\right) /(1+\cos 2 \alpha)$. This expression is minimized for $\alpha=0$, that is, for an orthonormal basis.
} 


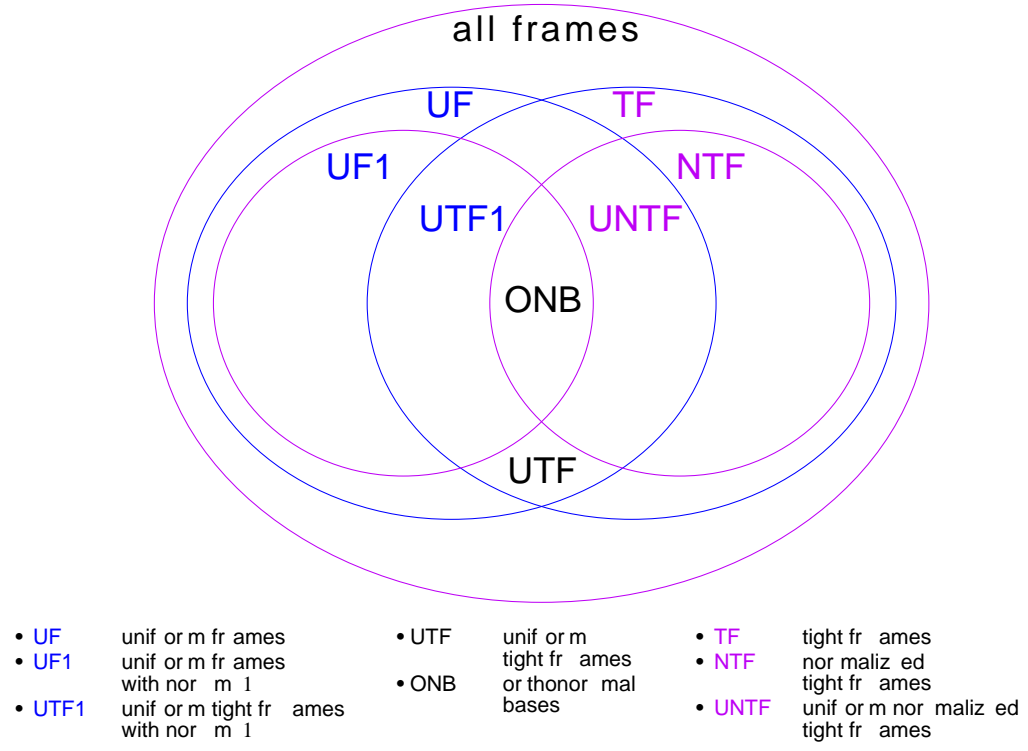

Fig. 3. Frames at a glance. Note that here we denote by UF uniform frames with the same norm, not necessarily 1 . When the norm is 1 , we say so explicitly, as in UF1, that is, uniform frames with norm 1.

The above expression is minimized when $\alpha=0$; back to the MB frame once more!

What we have done in this simple example is to show the types of issues that arise when trying to use frames to provide robustness. We have shown this when the frame elements belong to a finite-dimensional space such as $\mathbb{R}^{2}$, since the demonstrations are simple and the geometry intuitive. As mentioned previously, we extend these results to the frame elements from $\ell_{2}(\mathbb{Z})$; this simple example should serve as a guideline.

\section{Frame Expansions in $\mathbb{H}_{N}$ AND $\ell_{2}(\mathbb{Z})$}

We can now define frames more precisely. What we have seen in our simple example will generalize. We will find other frames with properties similar to those the MB frame possesses (such as conservation of energy).

A set of vectors $\Phi=\left\{\varphi_{i}\right\}_{i \in I}$ in a Hilbert space $H$, is called a frame if

$$
0<A\|x\|^{2} \leq \sum_{i \in I}\left|\left\langle\varphi_{i}, x\right\rangle\right|^{2} \leq B\|x\|^{2}<+\infty
$$

for all $x \neq 0$, where $I$ is the index set and the constants $A, B$ are called frame bounds. In this paper, we concentrate only on the $N$-dimensional real or complex Hilbert spaces $\mathbb{R}^{N}$ and $\mathbb{C}^{N}$ (which we denote $\mathbb{H}_{N}$ ) with the usual Euclidean inner product and on the Hilbert space of squaresummable sequences $H=\ell_{2}(\mathbb{Z})$ with the inner product $\langle x, y\rangle=\sum_{i \in I} x_{i}^{*} y_{i}$. For the former, $\Phi=\left\{\varphi_{i}\right\}_{i=1}^{M} \subset \mathbb{H}_{N}$ while for the latter, $\Phi=\left\{\varphi_{i}\right\}_{i \in \mathbb{Z}} \subset \ell_{2}(\mathbb{Z})$.

When $A=B$ the frame is tight (TF). If $A=B=1$, the frame is normalized tight (NTF). A frame is uniform (UF) if all its elements have norm $1,{ }^{6}\left\|\varphi_{i}\right\|=1$. For a UTF,

\footnotetext{
${ }^{6}$ Actually, the definition of a UF is more general; the norm is allowed to be $c \neq 1$. In this work, however, we consider only UF with norm
} 1. the frame bound $A$ gives the redundancy ratio (it is $3 / 2$ in our example). A UTF which is also normalized, that is, with $A=1$, is an orthonormal basis (ONB). Fig. 3 helps to clarify the "alphabet soup" of frames.

A frame operator $F$ maps the Hilbert space $H$ into $\ell_{2}(I)$

$$
(F x)_{i}=\left\langle\varphi_{i}, x\right\rangle,
$$

for $i \in I$. The frame operator can be represented by a matrix whose rows are the transposed frame vectors $\varphi_{i}^{*}$. When $H=\mathbb{H}_{N}$, the frame operator is an $M \times N$ matrix

$$
F=\left(\begin{array}{ccc}
\varphi_{11}^{*} & \cdots & \varphi_{1 N}^{*} \\
\vdots & \cdots & \vdots \\
\varphi_{M 1}^{*} & \cdots & \varphi_{M N}^{*}
\end{array}\right),
$$

while with $H=\ell_{2}(\mathbb{Z})$, the frame operator is an infinite matrix (infinite number of frame vectors and infinite number of elements in each vector). For the latter, we will examine a particular class of frames with vectors which are shifted versions of $M$ prototype ones. This will become clear in a moment.

The following theorem tells us that every tight frame can be seen as a projection of an orthonormal basis from a larger space.

Theorem 1 (Naimark [26]) ${ }^{7}$ A family $\left\{\varphi_{i}\right\}_{i \in I}$ in a Hilbert space $H$ is a normalized tight frame for $H$ if and only if there is a larger Hilbert space $H \subset K$ and an orthonormal basis $\left\{e_{i}\right\}_{i \in I}$ for $K$ so that the orthogonal projection $P$ of $K$ onto $H$ satisfies: $P e_{i}=\varphi_{i}$, for all $i \in I$.

${ }^{7}$ This theorem has been rediscovered by several people in recent years: The first author first heard it from I. Daubechies in the mid90 's. Han and Larson rediscovered it in [27]; they came up with the idea that a frame could be obtained by compressing a basis in a larger space and that the process is reversible. Finally, it was pointed out to the first author by E. Soljanin [29] that this is, in fact, Naimark's Theorem, which has been widely known in operator theory and has been used in quantum theory. The theorem was also proved in [28]. 


\section{A. Digression: Frame Interpretation of Filter Banks}

Fig. 1 depicts a signal-processing structure called a filter bank. It has been used extensively in compression as well as communications (with analysis and synthesis banks reversed) [33]. Early work in filter banks concentrated on trying to provide perfect reconstruction, that is, ensure that the output signal is only a shifted and possibly scaled version of the input signal. As the field matured, it was recognized that the filter bank implements a particular, structured linear transform [33]. Most of the research concentrated on critically-sampled filter banks, those with $M=N$, in which the filter impulse responses are basis functions from an orthogonal or a biorthogonal basis of $\ell_{2}(\mathbb{Z})$. Some researchers, however, tried to overcome certain critical sampling restrictions by oversampling, that is, by letting $M>N$ [34], [17], [18]. Which brings us to frames.

Have a look at Fig. 1 and assume that the filters $h_{i}[n], g_{i}[n], i=1, \ldots, M$, are all of length $N$. The input into the filter bank is a square-summable infinite sequence $x[n] \in \ell_{2}(\mathbb{Z})$. Let us now understand what such a filter bank is doing. The analysis filters act on $N$ samples at a time and then, due to downsampling by $N$, the same filters act on the following $N$ samples. In other words, there is no overlap. On the synthesis side, the reverse is true. This process is described by the following matrix equation:

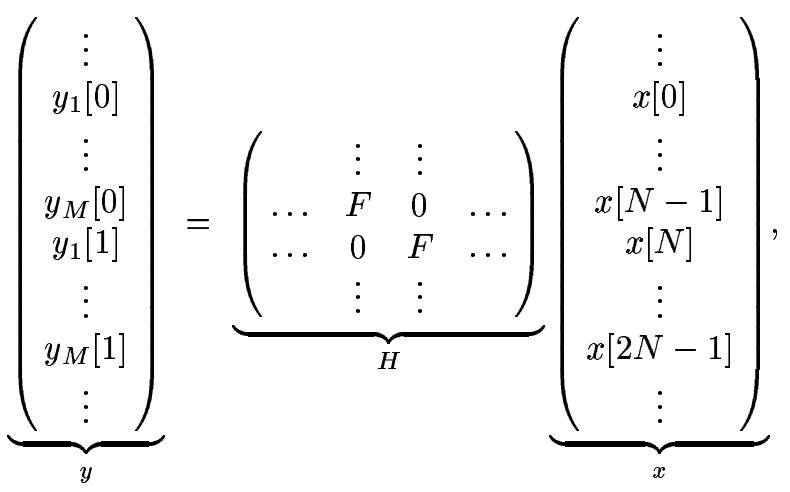

with

$$
F=\left(\begin{array}{ccc}
h_{1}[N-1] & \ldots & h_{1}[0] \\
\vdots & \ldots & \vdots \\
h_{M}[N-1] & \ldots & h_{M}[0]
\end{array}\right)
$$

Since the infinite matrix has a block-diagonal structure, we need only pay attention to the block $F$-an $M \times N$ matrix with time-reversed analysis filters' impulse responses as its rows. This rings a bell. In fact, the matrix $F$ is exactly a frame operator as described earlier in (7) and therefore, the filter bank as given in Fig. 1 implements a finite-dimensional frame expansion as we explained earlier. (Actually, the form of matrix $F$ is not all that we need; we still require the filters within to satisfy certain conditions to be explored later.) In other words

$$
\varphi_{i n}=h_{i}^{*}[N-n],
$$

for $i=1, \ldots, M$ and $n=1, \ldots, N$ (note that since $n$ is the time index, we number the elements of $h_{i}$ from 0 to
$N-1$ and those of $\varphi_{i}$ from 1 to $N$ ). Recall, however, that we restricted the filter length to be $N$, so there is no overlap. ${ }^{8}$ Lifting this restriction and allowing our filters to be of length larger than $N$ (though most of the time we will still require them to be of finite length-finite impulse response or FIR), brings us to the topic of this paper and explains why we restricted the frame vectors to be shifted versions of $M$ prototype ones. The $M$ prototypes are $M$ filters, and shifted versions arise due to the sliding convolution window and downsampling. The frame operator matrix $H$ is infinite, and although it possesses block structure, the blocks overlap. This prevents us from looking at a single block and forces us to find a simpler analysis method than dealing with infinite matrices.

We borrow the simpler method from the filter bank literature. Instead of looking at the infinite, time-domain matrix, we look at a so-called polyphase matrix $H(\omega)$ [33]. The polyphase matrix is based on gathering together samples whose time indices are congruent modulo $N$. This allows the system to be analyzed as time-invariant on vectors of length $N$.

For $i=1, \ldots, M, H_{i}(\omega)=\left[H_{i 1}(\omega), H_{i 2}(\omega), \ldots, H_{i N}(\omega)\right]^{*}$ is called the polyphase representation of the ith analysis fil$t e r^{9}$ where

$$
H_{i k}(\omega)=\sum_{n=-\infty}^{\infty} h_{i}[n N+k-1] e^{-j n \omega},
$$

are the polyphase components for $i=1, \ldots, M$ and $k=$ $1, \ldots, N$. To relate $H_{i k}(\omega)$ to a time-domain object, note that it is the discrete-time Fourier transform of the subsequence $h_{i}[n]$ obtained by retaining only the indices congruent to $k-1$ modulo $N$. Then $H(\omega)$ is the corresponding $M \times N$ analysis polyphase matrix with elements $H_{i k}(\omega)$. In other words, a polyphase decomposition is a decomposition into $N$ subsequences modulo $N$. When the filter length is $N$, then, each polyphase sequence is of length 1 . The polyphase matrix reduces to $H(\omega)=F J$, with $J$ an antidiagonal matrix ${ }^{10}$ that is, $H(\omega)$ becomes independent of $\omega$.

The following result establishes the equivalence between frames in $\ell_{2}(\mathbb{Z})$ and polyphase matrices with certain properties:

Proposition 1 (Cvetković and Vetterli [17]) A filter bank implements a frame decomposition in $\ell_{2}(\mathbb{Z})$ if and only if its analysis polyphase matrix is of full rank on the unit circle.

We now revisit briefly the definition of a UF. The frame is uniform if $\left\|h_{i}[n]\right\|=1$ for $i=1, \ldots, M$. Applying Parseval's relation to this condition, we get that

$$
1=\left\|h_{i}[n]\right\|^{2}=\frac{1}{2 \pi} \int_{-\pi}^{\pi}\left|H_{i}(\omega)\right|^{2} d \omega .
$$

\footnotetext{
${ }^{8}$ This is called a block transform in the filter bank literature. A block transform uses filters of length $N$ equal to the downsampling factor exactly as explained above. The whole procedure can be described by an infinite block-diagonal matrix as in (8).

${ }^{9}$ In the filter bank literature [33], this is usually the definition for the polyphase representation of the synthesis filter; we reverse the notation for convenience.

${ }^{10}$ The matrix $J$ just reverses the order of columns of $F$.
} 
Since shifted and upsampled polyphase components $e^{-j \omega k} H_{i k}(N \omega)$ and $e^{-j \omega l} H_{i l}(N \omega)$ are orthogonal (they do not overlap in time domain), the above expression is equal to

$$
\begin{aligned}
\frac{1}{2 \pi} \int_{-\pi}^{\pi}\left|H_{i}(\omega)\right|^{2} d \omega & =\frac{1}{2 \pi} \int_{-\pi}^{\pi} \sum_{k=1}^{N}\left|H_{i k}(N \omega)\right|^{2} d \omega \\
& =\frac{1}{2 \pi} \int_{-\pi}^{\pi} \sum_{k=1}^{N}\left|H_{i k}(\omega)\right|^{2} d \omega=1
\end{aligned}
$$

for $i=1, \ldots, M[35$, p. 52]. We used here the definition of a polyphase component (10) as well as periodicity.

Although many results generalize from finite dimensions to $\ell_{2}(\mathbb{Z})$, we need a more restricted definition of uniformity than what is available to us. This leads us to define strongly uniform frames:

Definition 1 (Strongly uniform frame) A frame expansion in $\ell_{2}(\mathbb{Z})$ implemented by an $M \times N$ polyphase matrix $H(\omega)$ is strongly uniform ${ }^{11}$ if

$$
\sum_{k=1}^{N}\left|H_{i k}(\omega)\right|^{2}=1,
$$

for $i=1, \ldots, M$ and $\omega \in[-\pi, \pi]$. This is equivalent to all the diagonal elements of $H(\omega) H^{*}(\omega)$ being 1 .

Clearly, strongly uniform frames are a subset of uniform frames. If $H(\omega)=F J$ and $F$ is uniform, then the corresponding frame is strongly uniform. Moreover, a square paraunitary matrix ${ }^{12}$ is automatically strongly uniform.

Further examples of strongly uniform frames will be shown later in this section.

In the remainder of this paper, we will use the frame operator $F$ in finite dimensions and polyphase matrix $H(\omega)$ when dealing with infinite sequences.

\section{B. Back to Frames}

After this filter bank interlude, let us go through certain important frame notions. Using the frame operator $F$, (5) can be rewritten as

$$
A I \leq F^{*} F \leq B I .
$$

It follows that $F^{*} F$ is invertible [16, Lemma 3.2.2], and furthermore

$$
B^{-1} I \leq\left(F^{*} F\right)^{-1} \leq A^{-1} I .
$$

Then, in finite dimensions, the dual frame of $\Phi$ is a frame defined as $\tilde{\Phi}=\left\{\tilde{\varphi}_{i}\right\}_{i=1}^{M}$, where

$$
\tilde{\varphi}_{i}=\left(F^{*} F\right)^{-1} \varphi_{i},
$$

for $i=1, \ldots, M$. Noting that $\tilde{\varphi}_{i}^{*}=\varphi_{i}^{*}\left(F^{*} F\right)^{-1}$ and stacking $\tilde{\varphi}_{1}^{*}, \tilde{\varphi}_{2}^{*}, \ldots, \tilde{\varphi}_{M}^{*}$ in a matrix, the frame operator associated with $\tilde{\Phi}$ is

$$
\tilde{F}=F\left(F^{*} F\right)^{-1} \text {. }
$$

\footnotetext{
${ }^{11}$ As before, when we say "strongly uniform", we will mean "strongly uniform with norm 1 ".

${ }^{12}$ A square matrix $H(\omega)$ is called paraunitary if $H^{*}(\omega) H(\omega)=$ $H(\omega) H^{*}(\omega)=c I, c \neq 0$.
}

Since $\tilde{F}^{*} \tilde{F}=\left(F^{*} F\right)^{-1},(13)$ shows that $B^{-1}$ and $A^{-1}$ are frame bounds for $\tilde{\Phi}$.

Another important concept is that of a pseudo-inverse $F^{\dagger}$. It is the frame operator associated with the dual frame,

$$
F^{\dagger}=\tilde{F}^{*} .
$$

Similarly, for infinite sequences, the dual frame is represented by

$$
\tilde{H}(\omega)=H(\omega)\left(H^{*}(\omega) H(\omega)\right)^{-1},
$$

while the pseudo-inverse is

$$
H^{\dagger}(\omega)=\tilde{H}^{*}(\omega)
$$

Note that for any matrix $F$ with rows $\varphi_{i}^{*}$

$$
F^{*} F=\sum_{i=1}^{M} \varphi_{i} \varphi_{i}^{*} .
$$

This identity will prove to be useful in many proofs.

\section{The Role of Eigenvalues}

The products $F^{*} F$ and $H^{*}(\omega) H(\omega)$ will appear everywhere; their eigenstructures play an important role. Denote by $\lambda_{k}$ 's the eigenvalues of $F^{*} F$ and by $\lambda_{k}(\omega)$ 's the spectral eigenvalues of $H^{*}(\omega) H(\omega)$, where a spectral eigenvalue for a fixed $\omega_{0}$ is the eigenvalue of $H^{*}\left(\omega_{0}\right) H\left(\omega_{0}\right)$. We could, of course, just analyze the infinite case and then specialize it to finite dimensions with $H(\omega)=F J$ when needed. However, we keep the discussions separate for clarity. We now summarize important eigenvalue properties.

\section{C.1 General Frame}

For any frame in $\mathbb{H}_{N}$, the sum of the eigenvalues of $F^{*} F$, equals the sum of the lengths of the frame vectors:

$$
\sum_{k=1}^{N} \lambda_{k}=\sum_{i=1}^{M}\left\|\varphi_{i}\right\|^{2} .
$$

For $H=\ell_{2}(\mathbb{Z})$, the integral sum of the spectral eigenvalues of $H^{*}(\omega) H(\omega)$ equals the sum of the filters' norms:

$$
\frac{1}{2 \pi} \int_{-\pi}^{\pi} \sum_{k=1}^{N} \lambda_{k}(\omega) d \omega=\sum_{i=1}^{M}\left\|h_{i}[n]\right\|^{2} .
$$

\section{C.2 Uniform Frame}

For a uniform frame, that is, when $\left\|\varphi_{i}\right\|=1, i=$ $1, \ldots, M$,

$$
\sum_{k=1}^{N} \lambda_{k}=\sum_{i=1}^{M}\left\|\varphi_{i}\right\|^{2}=M .
$$

Not surprisingly, the integral sum of spectral eigenvalues equals $M$ as well,

$$
\frac{1}{2 \pi} \int_{-\pi}^{\pi} \sum_{k=1}^{N} \lambda_{k}(\omega) d \omega=M .
$$




\section{C.3 Tight Frame}

Since tightness means $A=B$, for a $\mathrm{TF}$, we have from (5)

$$
\sum_{i=1}^{M}\left|\left\langle f, \varphi_{i}\right\rangle\right|^{2}=A\|f\|^{2}
$$

for all $f \in H_{N}$. Moreover, according to (13), a frame is a $\mathrm{TF}$ if and only if

$$
F^{*} F=A \cdot I_{N} .
$$

Thus, for a TF, all the eigenvalues of $F^{*} F$ are equal to $A$. Then, using (20), the sum of the eigenvalues of $F^{*} F$ is as follows:

$$
N \cdot A=\sum_{k=1}^{N} \lambda_{k}=\sum_{i=1}^{M}\left\|\varphi_{i}\right\|^{2} .
$$

If we are dealing with infinite sequences, analogous results can be formulated. The following is known:

Proposition 2 (Cvetković and Vetterli [17]) A filter bank implements a tight frame expansion in $\ell_{2}(\mathbb{Z})$ if and only if $H^{*}(\omega) H(\omega)=A I_{N}$.

Proposition 3 (Vaidyanathan [36]) An $M \times N$ polyphase matrix $H(\omega)$ represents a tight frame if and only if it has the following decomposition:

$$
H(\omega)=U(\omega) F,
$$

where $U(\omega)$ is an $M \times M$ paraunitary matrix ${ }^{13}$ and $F$ is an $M \times N$ matrix such that $F^{*} F=A I_{N}$, that is, $F$ is a tight frame operator.

Proposition 4 (Cvetković [35, Thm. 7]) For a frame associated with an FIR filter bank, with the polyphase analysis matrix $H(\omega)$, its dual frame (17) consists of finite length vectors if and only if $H^{*}(\omega) H(\omega)$ is unimodular. ${ }^{14}$

This result leads us to formulate the following useful property of TFs:

Corollary 1: Given an FIR analysis polyphase matrix $H(\omega)$ corresponding to a $\mathrm{TF}$, the synthesis polyphase matrix $G(\omega)$ corresponding to the pseudo-inverse as in (18) is FIR as well.

Using Proposition 2, we know that $H^{*}(\omega) H(\omega)=A I_{N}$. Since $H(\omega)$ is FIR, $H(\omega) / \sqrt{A}$ is FIR as well. Thus $(H(\omega) / \sqrt{A})^{*}(H(\omega) / \sqrt{A})=I_{N}$ is unimodular. By Proposition 4 , the dual frame (synthesis polyphase matrix) to $H(\omega) / \sqrt{A}$ is FIR as well. Since scaling does not affect the FIR property, the dual frame (synthesis polyphase matrix) to $H(\omega)$ is FIR.

As for the eigenvalues, $H^{*}(\omega) H(\omega)$ has eigenvalues constant over the unit circle and equal to $A$ with multiplicity $N$, that is, for $k=1, \ldots, N$ :

$$
\lambda_{k}(\omega)=A .
$$

\footnotetext{
${ }^{13}$ Moreover, any paraunitary matrix can be decomposed into a sequence of elementary matrices such as rotations and delays [36].

${ }^{14}$ Here unimodular means that the determinant of $H^{*}(\omega) H(\omega)$ is \pm 1 .
}

\section{C.4 Normalized Tight Frame}

If a frame is an NTF, that is, $A=B=1$, then

$$
\sum_{i=1}^{M}\left|\left\langle f, \varphi_{i}\right\rangle\right|^{2}=\|f\|^{2}
$$

for all $f \in H_{N}$. In operator notation, a frame is an NTF if and only if

$$
F^{*} F=I_{N} .
$$

For an NTF, all the eigenvalues of $F^{*} F$ are equal to 1 .

Then, using (20), the sum of the eigenvalues of $F^{*} F$ is as follows:

$$
N=\sum_{k=1}^{N} \lambda_{k}=\sum_{i=1}^{M}\left\|\varphi_{i}\right\|^{2} .
$$

The same is, of course, true for an NTF in $H=\ell_{2}(\mathbb{Z})$.

\section{C.5 Uniform Tight Frame}

From (22) and (26), we see that

$$
N \cdot A=\sum_{k=1}^{N} \lambda_{k}=\sum_{i=1}^{M}\left\|\varphi_{i}\right\|^{2}=M .
$$

Then, from (24) and (30),

$$
\sum_{i=1}^{M}\left|\left\langle f, \varphi_{i}\right\rangle\right|^{2}=\frac{M}{N}\|f\|^{2}
$$

for all $f \in H_{N}$. The redundancy ratio is then

$$
A=\frac{M}{N} \text {. }
$$

Since $F^{*} F=(M / N) I$, the following is obvious:

$$
\sum_{i=1}^{M}\left|\varphi_{i k}\right|^{2}=\frac{M}{N} .
$$

The same is true for sequences, that is, $H^{*}(\omega) H(\omega)$ has eigenvalues constant over the unit circle and equal to $M / N$ with multiplicity $N$. Similarly to (33), we see that

$$
\sum_{i=1}^{M}\left|H_{i k}(\omega)\right|^{2}=\frac{M}{N}
$$

\section{C.6 Uniform Normalized Tight Frame}

If a frame is a UNTF, that is, we also ask for $A=B=1$, then

$$
N=\sum_{k=1}^{N} \lambda_{k}=\sum_{i=1}^{M}\left\|\varphi_{i}\right\|^{2}=M,
$$

and thus, a UNTF is an orthonormal basis. 


\section{Examples of Uniform and Strongly Uniform Frames}

Oversampled filter banks are sometimes preferred to classical critically sampled filter banks for their greater design freedom. However, this freedom makes the actual design difficult.

One of the most used families of oversampled filter banks are nondownsampled filter banks. They are obtained by eliminating the downsampling in the filter bank scheme. If the analysis and synthesis filters are power complementary (that is, with FIR filters, up to a scaling factor, the synthesis filters are the time-reversed versions of the analysis ones) then the corresponding frame is tight and uniform but not strongly uniform.

It will be shown in next sections that strongly uniform tight frames constitute an important class of frames. We propose the following factorization to design polyphase matrices corresponding to strongly uniform tight frames:

$$
H(\omega)=F U(\omega),
$$

where $F$ is an $M \times N$ uniform tight frame in $\mathbb{H}_{N}$ and $U(\omega)$ is an $N \times N$ paraunitary matrix. It is easy to see that such a polyphase matrix corresponds to a strongly uniform tight frame.

Note the difference between this factorization and the one in Proposition $3(H(\omega)=U(\omega) F)$. The order of the elements is reversed, so in this last factorization, the paraunitary matrix has size $M \times M$, while in our factorization it has size $N \times N(N<M)$. This is not surprising since the family of polyphase matrices with the factorization $H(\omega)=U(\omega) F$ represents a more general class of tight frames and not the restricted class of strongly uniform tight frames.

We cannot claim that our factorization includes all possible strongly uniform tight frames; however, the following is true:

Theorem 2: Define an equivalence relation by bundling a frame implemented with an FIR oversampled filter bank with all frames that result from rigid rotations or reflections of the entire frame as well as negations or shifts of some individual elements (that is, $h_{i}[n] \rightarrow-h_{i}[n-k] k \in \mathbb{Z}$ ). When $M=N+1$, there is a single equivalence class for all strongly uniform tight frames.

Proof: See Appendix A-A.

Since a UTF $F$ in $\mathbb{H}_{N}$ can be seen as a strongly uniform tight frame in $\ell_{2}(\mathbb{Z})$ (that is, $H(\omega)=F J$ ), Theorem 2 basically says that the factorization in (35) includes all the possible strongly uniform tight frames when $M=N+1$ (up to a shift or negation of some individual elements). Also, when $H(\omega)=F J$, this theorem reduces to Theorem 2.6 from $[8]$.

For example, the MB tight frame from our simple example describes all possible UTFs with $N=2$ and $M=3$ in finite dimensions; the same is true for sequences, that is, the factorization $H(\omega)=F U(\omega)$ with $F$ the MB frame, describes all possible SUTFs with $N=2$ and $M=3$.

Unfortunately, when $M$ exceeds $N+1$, there are uncountably many equivalence classes of the type described above; thus, we cannot systematically obtain all uniform tight frames. However, at least for $N=2$, UTFs still have a simple characterization.

Theorem 3 (Goyal, Kovačević and Kelner [8, Thm. 2.7]) The following are equivalent:

1. $\left\{\varphi_{k}=\left(\cos \alpha_{k}, \sin \alpha_{k}\right)\right\}_{k=1}^{M}$ is a uniform tight frame.

2. $\sum_{k=1}^{M} z_{k}=0$ where $z_{k}=e^{j 2 \alpha_{k}}$ for $k=1,2, \ldots, M$.

Thus, a simple combination of our factorization (35) together with the complete characterization of UTFs for $N=2$ given by the above theorem, produces a useful (although probably not complete) factorization of SUTFs.

\section{E. Harmonic Frames}

We now turn our attention to an important family of frames-harmonic tight frames (HTF). These frames are obtained by keeping the first $N$ coordinates of an $M \times M$ discrete Fourier transform basis. They will prove to be useful for our application.

A complex HTF is given by:

$$
\varphi_{i k}=\frac{1}{\sqrt{N}} W_{M}^{(i-1)(k-1)}
$$

for $k=1, \ldots, N$ and $i=1, \ldots, M$, where $W_{M}=e^{j 2 \pi / M}$. A real HTF could be defined similarly [8]. A more general definition of the harmonic frame (general harmonic frame) is given in [37].

As a direct consequence of Theorem 2, we see that any UTF with $M=N+1$ is equivalent to the HTF with $M=$ $N+1$. This is a very useful result since we have HTFs for any $N$ and $M$; thus, for $M=N+1$, we always have an expression for all UTFs.

Another interesting property of an HTF is that it is the only NTF with equal-norm elements which are generated by a group of unitary operators with one generator, that is, $\Phi=\left\{\varphi_{i}\right\}_{i=1}^{M}=\left\{U^{i} \varphi_{0}\right\}_{i=1}^{M}$, where $U$ is a unitary operator [37], [38].

Moreover, HTFs have a very convenient property when it comes to erasures. We can erase any $e \leq(M-N)$ elements from the original frame; what is left is still a frame [8, Thm. 4.2]. This will be extended in Section V-A to frames represented by $H(\omega)=F U(\omega)$ where $F$ is an HTF (Theorem 6).

\section{Quantized Frame Expansions in $\mathbb{H}_{N}$ And $\ell_{2}(\mathbb{Z})$}

In this section we will analyze the effect of quantization under a very simple model. For the moment we assume that there are no erasures during transmission. We want the reconstruction operator to be linear, that is, we want it to be implemented by a synthesis filter bank. The reconstruction operator that we will use is the pseudo-inverse (18).

We will assume that the quantization error can be treated as additive white noise with variance $\sigma^{2}=\Delta^{2} / 12$, where $\Delta$ represents the step size of the quantizer and each quantizer has the same step size. We further assume that the noise sequences generated by two different channels are 
pairwise uncorrelated. This can be expressed as:

$$
\hat{y}_{i}[n]=y_{i}[n]+w_{i}[n],
$$

for $i=1, \ldots, M$, and

$$
E\left[w_{i}[n] w_{j}^{*}[n-m]\right]=\sigma^{2} \delta_{i j} \delta[m] .
$$

Now comes the justification of a pseudo-inverse. Under this assumption (input sequences corrupted by additive white noise), the pseudo-inverse in (16) is the best linear reconstruction operator in the mean square sense [16]. The same could be shown for (18). Moreover, in Appendix A-B we show that the MSE due to quantization is:

$$
\begin{aligned}
\mathrm{MSE} & =\frac{\sigma^{2}}{2 \pi N} \int_{-\pi}^{\pi} \operatorname{tr}\left(\left(H^{*}(\omega) H(\omega)\right)^{-1}\right) d \omega \\
& =\frac{\sigma^{2}}{2 \pi N} \int_{-\pi}^{\pi} \sum_{k=1}^{N} \frac{1}{\lambda_{k}(\omega)} d \omega,
\end{aligned}
$$

where $\lambda_{k}(\omega), k=1, \ldots, N$ denote the spectral eigenvalues of $H^{*}(\omega) H(\omega)$. We will be using the above two expressions interchangeably. Recall that the integral sum of the eigenvalues is constant and if we are encoding with a uniform frame, it is equal to $M$. Thus, we want to minimize the MSE given the constraint that the integral sum of the eigenvalues is constant. This occurs when the eigenvalues are equal and constant over $\omega$ which is true if and only if the original frame is tight. We can then state the following theorem:

Theorem 4: When encoding with a filter bank implementing a uniform frame and decoding with the pseudoinverse under the noise model (37)-(38), the MSE is minimum if and only if the frame is tight. Then:

$$
\mathrm{MSE}_{0}=\frac{N}{M} \sigma^{2} .
$$

This optimality of TFs among UFs holds also for $H=$ $\mathbb{H}_{N}[8]$. This makes sense, since the only difference in the expression for the MSE given by (40) is that the eigenvalues depend on $\omega$. Since the proof of the theorem is essentially the same as the corresponding proof in [8], we omit it here.

\section{A. A Note on Linear Reconstruction}

We have assumed the use of a linear reconstruction algorithm. In the implausible case that the input and output of each quantizer are jointly Gaussian, linear reconstruction is necessarily optimal. Otherwise, some nonlinear estimate will generally be better, but determining such an estimate requires knowledge of the input signal distribution and is computationally difficult. Of particular present concern is that a simple and explicit reconstruction algorithm facilitates the analysis and optimization of the system.

One alternative to linear reconstruction is called consistent reconstruction. Consistent reconstruction is based on viewing the encoder (analysis filter bank and quantization) as partitioning the input signal space. Any estimate in the same partition cell as the true signal will produce the same quantized output and hence is said to be "consistent" with the true signal. Consistent estimates depend on the filter bank and quantizers, but not on the input signal distribution. Nevertheless, in many scenarios consistent reconstruction performs within a constant factor of optimal reconstruction while linear reconstruction is much worse [20], [39], [40], [8], [21]. Empirical evidence presented in [8] suggests that the MSE under the assumption of linear reconstruction is a reasonable objective function even if consistent reconstruction is used.

\section{Introducing Erasures}

Here we consider the effect of erasures on the structure of the frame and on the MSE. We denote by $E$ the index set of erasures and by $H_{E}(\omega)$ the polyphase matrix after $e=|E|$ erasures. $H_{E}(\omega)$ is an $(M-e) \times N$ matrix obtained by deleting the $E$-numbered rows from the $M \times N$ polyphase matrix $H(\omega)$. The first question to be answered is under which conditions $H_{E}(\omega)$ still represents a frame. We then study the effect of erasures on the MSE.

It is interesting to note that there are families of frames for which the properties of the frame after erasures do not depend on the actual frame element removed. An example is the class of geometrically uniform frames [41].

\section{A. Effect of Erasures on the Structure of a Frame}

Our aim is to use the pseudo-inverse of $H_{E}(\omega)$ to reconstruct after $e$ erasures. The pseudo-inverse matrix is defined only if the matrix $H_{E}(\omega)$ still represents a frame, that is, if and only if it is still of full rank on the unit circle. This leads to the following definition:

Definition 2: An oversampled filter bank which implements a frame expansion represented by a polyphase matrix $H(\omega)$ is said to be robust to e erasures when for any erasure set $E$ with $|E|=e, H_{E}(\omega)$ is of full rank on the unit circle.

Let us consider first the case where there is only one erasure.

Theorem 5: An oversampled filter bank which implements a uniform tight frame is robust to one erasure if and only if

$$
\sum_{k=1}^{N}\left|H_{i k}(\omega)\right|^{2}<\frac{M}{N},
$$

for $i=1, \ldots, M$ and for all $\omega$.

Proof: See Appendix A-C.

Recall that with an SUTF: $\sum_{k=1}^{N}\left|H_{i k}(\omega)\right|^{2}=1$, for $i=1, \ldots, M$ and for all $\omega$. In finite dimensions, a UTF is always robust to one erasure $[8, \mathrm{Thm} .4 .1]$. This is easily seen from the above theorem if we substitute $H(\omega)=$ $F J$, that is, $H_{i k}(\omega)=h_{i}[k-1]$ and $\sum_{k=1}^{N}\left|H_{i k}(\omega)\right|^{2}=$ $\sum_{k=1}^{N}\left|h_{i}[k-1]\right|^{2}=1<M / N$, since it is a uniform frame and $\left\|h_{i}\right\|=1$.

A consequence of the previous theorem is:

Corollary 2: Any oversampled filter bank which implements a strongly uniform tight frame is robust to one erasure. 
Theorem 5 does not reveal anything about the existence of filter banks that are robust to more than one erasure. However, it has been shown that an HTF in $\mathbb{H}_{N}$ is robust to $M-N$ erasures [8]. This can be used to show the existence of a family of SUTFs in $\ell_{2}(\mathbb{Z})$ that are robust to $e$ erasures for $e \leq M-N$.

Theorem 6: Consider an oversampled filter bank with a polyphase matrix $H(\omega)=F U(\omega)$, where $F$ is an HTF in $\mathbb{H}_{N}$, and $U(\omega)$ is an $N \times N$ polyphase matrix nonsingular on the unit circle $(\operatorname{det}(U(\omega)) \neq 0)$. This filter bank is robust to $e$ erasures $(e \leq M-N)$.

Proof: See Appendix A-D.

If $U(\omega)$ is a paraunitary matrix, the resulting oversampled filter bank $H(\omega)=F U(\omega)$ represents an SUTF robust to $e$ erasures $(e \leq M-N)$.

\section{B. Effect of Erasures on the MSE}

In the previous section, it was shown that it is possible to design oversampled filter banks which are robust up to $M-N$ erasures. We assume such filter banks for the rest of the paper.

Now, we want to compute the effect of the erasures on the MSE. Call $H(\omega)$ the polyphase matrix related to the original frame and $H_{E}(\omega)$ the polyphase matrix after $e=|E|$ erasures. The reconstruction uses the dual polyphase matrix $H_{E}^{\dagger}(\omega)$ and the quantization model is the one proposed in (37)-(38). Under these assumptions the mean square error is equivalent to the one determined in (39)-(40):

$$
\begin{aligned}
\operatorname{MSE}_{E} & =\frac{\sigma^{2}}{2 \pi N} \int_{-\pi}^{\pi} \operatorname{tr}\left(\left(H_{E}^{*}(\omega) H_{E}(\omega)\right)^{-1}\right) d \omega \\
& =\frac{\sigma^{2}}{2 \pi N} \sum_{k=1}^{N} \int_{-\pi}^{\pi} \frac{1}{\lambda_{k}\left(H_{E}^{*}(\omega) H_{E}(\omega)\right)} d \omega,
\end{aligned}
$$

where $\lambda_{k}\left(H_{E}^{*}(\omega) H_{E}(\omega)\right)$, for $k=1, \ldots, N$, are the spectral eigenvalues of $H_{E}^{*}(\omega) H_{E}(\omega)$.

However, our target is to express the mean square error in terms of the original frame and to find properties that the original frame operator has to satisfy to minimize the distortion. Consider first a strongly uniform frame and $e=1$ :

Theorem 7: Consider encoding with a strongly uniform frame and decoding with linear reconstruction. The MSE averaged over all possible erasures of one channel is minimum if and only if the original frame is tight. Moreover, a tight frame minimizes the maximum distortion caused by one erasure. The MSE is given by:

$$
\mathrm{MSE}_{1}=\left(1+\frac{1}{M-N}\right) \mathrm{MSE}_{0}
$$

where $\mathrm{MSE}_{0}$ is given by (41).

Proof: See Appendix A-E.

It is hard to extend this theorem to cases with more than one erasure. However, it is possible to compute the MSE with $e>1$ when the original frame is strongly uniform and tight:

$$
\mathrm{MSE}_{E}=\left(1+\frac{1}{2 \pi} \int_{-\pi}^{\pi} \sum_{k=1}^{e} \frac{\mu_{k}(\omega)}{M-N \mu_{k}(\omega)} d \omega\right) \mathrm{MSE}_{0}
$$

where $\mu_{k}(\omega)$ are the spectral eigenvalues of $T^{*}(\omega) T(\omega)$ and $T(\omega)$ is the $N \times e$ polyphase matrix of erased components with columns $\left\{H_{i}(\omega)\right\}_{i \in E}$. The derivation of (45) follows closely that for $H=\mathbb{H}_{N}$ in [8], so we omit it here.

Note first that with one erasure, $T^{*}(\omega) T(\omega)=$ $H_{i}^{*}(\omega) H_{i}(\omega)=1$ and thus the single eigenvalue $\mu(\omega)=1$, reducing (45) to (44).

Expression (45) is similar to (40), and the spectral sum of the $e$ eigenvalues of $T(\omega)$ is constrained to be a constant, that is, $\sum_{k=1}^{e} \mu_{k}(\omega)=\operatorname{tr}\left(T^{*}(\omega) T(\omega)\right)=e$. Thus, the minimum in (45) occurs when all the eigenvalues are equal to 1 if possible.

If $e \leq N$, it is indeed possible to have $\mu_{k}(\omega)=1$, for $i=1, \ldots, e$. This occurs if and only if the erased vectors are pairwise orthogonal. Then $T^{*}(\omega) T(\omega)=I_{e}$ and (45) gives

$$
\mathrm{MSE}_{e \text { orthogonal erasures }}=\left(1+\frac{e}{M-N}\right) \mathrm{MSE}_{0} .
$$

If $e>N$, it is not possible to have $e$ eigenvalues equal to 1 because there will be at most $N$ nonzero eigenvalues in the $N \times N$ matrix $T^{*}(\omega) T(\omega)$. Denoting the nonzero eigenvalues $\left\{\mu_{k}(\omega)\right\}_{k=1}^{N}$,

$$
\mathrm{MSE}_{E}=\left(1+\frac{1}{2 \pi} \int_{-\pi}^{\pi} \sum_{k=1}^{N} \frac{\mu_{k}(\omega)}{M-N \mu_{k}(\omega)} d \omega\right) \mathrm{MSE}_{0} .
$$

This MSE is minimized when $\mu_{k}(\omega)=e / N, i=1, \ldots, N$, which occurs when the erased elements form a tight frame. When any erasure event is possible-meaning any combination of switches may be open in Fig. 1-it is not possible to make $T(\omega)$ always correspond to a tight frame. There are situations in which the number of "physical" channels (separate transmission media) is less than the number of branches in the analysis filter bank. In this case, there may be sets of channels that are each completely lost or completely received and then it may be possible for the erased vectors to form a tight frame.

\section{Concluding Remarks}

Given the recent surge of interest in frames and their applications, we continued the previous work [8] of two of the authors where frames are elements of $\mathbb{R}^{N}$ or $\mathbb{C}^{N}$. In this work, we allowed our frame elements to be from $\ell_{2}(\mathbb{Z})$. Moreover, we require these frames to have a filter bank implementation.

We investigated the robustness of such frames to erasures after quantization. We found that any UTF is optimal when no erasures are present (Theorem 4). When there is one erasure, we know that any oversampled filter bank which implements an SUTF is robust to one erasure (Theorem 5) and minimizes the MSE (Theorem 7). When there 
are $e>1$ erasures, depending on whether $e$ is smaller or larger then $N$, the minimum in (45) occurs when the erased elements are either orthogonal or form a tight frame.

The results in this paper thus present what is known to date about frames which have a filter bank implementation when subjected to erasures. Some related issues include classification of UTF robust to particular sets of erasures [37] and finding other frame families with properties similar to those HTFs such as efficient computation and robustness to erasures. Moreover, we are investigating the use of frames in multiple-antenna wireless systems [42].

\section{APPENDIX}

\section{Proofs}

\section{A. Proof of Theorem 2}

Given a strongly uniform tight frame represented by the polyphase matrix $K(\omega)$, all the other polyphase matrices related to the same equivalent class are obtained as follows:

$$
H(\omega)=\Sigma K(\omega) U(\omega),
$$

where $U(\omega)$ is an $N \times N$ paraunitary matrix, $\Sigma=$ $\operatorname{diag}\left(\sigma_{1}, \sigma_{2}, \ldots, \sigma_{M}\right)$ and $\sigma_{i}= \pm e^{-j l \omega}, l \in \mathbb{Z}, i=$ $1, \ldots, M$. This equivalence class preserves tightness, uniformity and strong uniformity. Thus, if $K(\omega)$ is strongly uniform and tight, so is $H(\omega)$.

Now, let $H(\omega)$ be a polyphase matrix associated with an SUTF with $M=N+1$. It can be shown that it consists of the first $N$ columns of a scaled $(N+1) \times(N+1)$ paraunitary matrix $\bar{H}(\omega)$. Each row (or column) of $\bar{H}(\omega)$ is of norm $\sqrt{(N+1) / N}$, that is:

$$
\sum_{k=1}^{N+1}\left|\bar{H}_{i k}(\omega)\right|^{2}=\frac{N+1}{N},
$$

for $i=1,2, \ldots, N+1$. Moreover, since our frame is strongly uniform we have:

$$
\sum_{k=1}^{N}\left|\bar{H}_{i k}(\omega)\right|^{2}=1
$$

for $i=1,2, \ldots, N+1$. Subtracting (48) from (47) we obtain:

$$
\left|\bar{H}_{i, N+1}(\omega)\right|^{2}=\frac{1}{N} .
$$

Since $H(\omega)$ is realized with FIR filters, it is formed only of Laurent polynomial elements. This implies that $\bar{H}_{i, N+1}(\omega)$ must be a monomial : $\bar{H}_{i, N+1}(\omega)= \pm N^{-1 / 2} e^{-j l \omega}$ $l \in \mathbb{Z}$. Without loss of generality we assume that $\bar{H}_{i, N+1}(\omega)= \pm N^{-1 / 2}$. That is, the last column of $\bar{H}(\omega)$ is $\left( \pm N^{-1 / 2}, \pm N^{-1 / 2}, \ldots, \pm N^{-1 / 2}\right)$ for some choice of signs.

Any given choice of signs in $\bar{H}_{i, N+1}(\omega)$ determines a subspace. Thus the span of the other $N$ subspaces (each subspace is related to one of the channels) must be the orthogonal complement to this subspace. Since orthonormal bases for a subspace are unitarily equivalent, the possible tight frames corresponding to a single choice of signs are in the same equivalence class. Flipping signs yields frames in the same equivalence class.

\section{B. Derivation of (40)}

We now find the error of the reconstruction after the frame coefficients have been quantized:

$$
\begin{aligned}
\mathrm{MSE} & =E\left[\|x[n]-\hat{x}[n]\|^{2}\right] \\
& =\frac{1}{2 \pi N} \int_{-\pi}^{\pi} E\left[\|X(\omega)-\hat{X}(\omega)\|^{2}\right] d \omega \\
& =\frac{1}{2 \pi N} \int_{-\pi}^{\pi} E\left[\left(H^{\dagger}(\omega) W(\omega)\right)^{*}\left(H^{\dagger}(\omega) W(\omega)\right)\right] d \omega \\
& =\frac{1}{2 \pi N} \int_{-\pi}^{\pi} E\left[\sum_{i, j=1}^{M} W_{i}^{*}(\omega) W_{j}(\omega) \tilde{H}_{i}^{*}(\omega) \tilde{H}_{j}(\omega)\right] d \omega \\
& =\frac{\sigma^{2}}{2 \pi N} \int_{-\pi}^{\pi} \sum_{i=1}^{M} \tilde{H}_{i}^{*}(\omega) \tilde{H}_{i}(\omega) d \omega \\
& =\frac{\sigma^{2}}{2 \pi N} \int_{-\pi}^{\pi} \operatorname{tr}\left(\tilde{H}^{*}(\omega) \tilde{H}(\omega)\right) d \omega \\
& =\frac{\sigma^{2}}{2 \pi N} \int_{-\pi}^{\pi} \operatorname{tr}\left(\left(H^{*}(\omega) H(\omega)\right)^{-1}\right) d \omega \\
& =\frac{\sigma^{2}}{2 \pi N} \int_{-\pi}^{\pi} \sum_{k=1}^{N} \frac{1}{\lambda_{k}(\omega)} d \omega .
\end{aligned}
$$

\section{Proof of Theorem 5}

Assume that the erased channel is $H_{i}(\omega)$. Call $H_{\{i\}}(\omega)$ the polyphase matrix after one erasure. Using (19), we get

$$
\begin{aligned}
H_{\{i\}}^{*}(\omega) H_{\{i\}}(\omega) & =H^{*}(\omega) H(\omega)-H_{i}(\omega) H_{i}^{*}(\omega)(49) \\
& =\frac{M}{N} I_{N}-H_{i}(\omega) H_{i}^{*}(\omega) .
\end{aligned}
$$

$H_{\{i\}}(\omega)$ is a frame if and only if $H_{\{i\}}^{*}(\omega) H_{\{i\}}(\omega)$ is of full rank on the unit circle. That means that $\left(H_{\{i\}}^{*}(\omega) H_{\{i\}}(\omega)\right)^{-1}$ must exist on the unit circle. The identity:

$$
(A-B C D)^{-1}=A^{-1}+A^{-1} B\left(C^{-1}-D A^{-1} B\right)^{-1} D A^{-1},
$$

with $A=(M / N) I_{N}, B=H_{i}(\omega), C=1$, and $D=H_{i}^{*}(\omega)$ yields:

$$
\begin{aligned}
& \left(H_{\{i\}}^{*}(\omega) H_{\{i\}}(\omega)\right)^{-1} \\
= & \frac{N}{M} I_{N} \\
+ & \frac{N}{M} I_{N} H_{i}(\omega)\left(1-H_{i}^{*}(\omega) \frac{N}{M} I_{N} H_{i}(\omega)\right)^{-1} H_{i}^{*}(\omega) \frac{N}{M} I_{N} \\
= & \frac{N}{M} I_{N}+\frac{N^{2}}{M^{2}}\left(1-\frac{N}{M} H_{i}^{*}(\omega) H_{i}(\omega)\right)^{-1} H_{i}(\omega) H_{i}^{*}(\omega) .
\end{aligned}
$$

Thus, the matrix is invertible if and only if:

$$
1-\frac{N}{M} H_{i}^{*}(\omega) H_{i}(\omega) \neq 0
$$

for all $\omega$. The desired inequality now follows from the fact that the frequency response of each filter is continuous (since we are only considering FIR filters) and the 
frame is uniform. The continuity of the filters implies that $\sum_{k=1}^{N}\left|H_{i k}(\omega)\right|^{2}<M / N$, for all $\omega$ or $\sum_{k=1}^{N}\left|H_{i k}(\omega)\right|^{2}>$ $M / N$, for all $\omega$. However, since the frame is uniform, that is, $(1 / 2 \pi) \int_{-\pi}^{\pi} \sum_{k=1}^{N}\left|H_{i k}(\omega)\right|^{2}=1$, then $\sum_{k=1}^{N}\left|H_{i k}(\omega)\right|^{2}<$ $M / N$, for all $\omega$.

\section{Proof of Theorem 6}

First note that if a finite set of channels has a subset that is a frame, then the original set of channels is also a frame. Thus it suffices to consider subsets with $N$ channels; since all of these will be shown to be frames, larger subsets are also frames.

Let us call $H_{E}(\omega)$ the $N \times N$ polyphase matrix after $e=M-N$ erasures. $H_{E}(\omega)$ is a frame if and only if $\operatorname{det}\left(H_{E}(\omega)\right) \neq 0$ on the unit circle. Now, we know that $\operatorname{det}\left(F_{E}\right) \neq 0$ for any subset of $e=(M-N)$ erasures [8] and since: $H_{E}(\omega)=F_{E} U(\omega)$,

$$
\operatorname{det}\left(H_{E}(\omega)\right)=\operatorname{det}\left(F_{E}\right) \operatorname{det}(U(\omega)) \neq 0
$$

for all $\omega$.

\section{E. Proof of Theorem 7}

As in the proof of Theorem 5, assume that the erased channel is $H_{i}(\omega)$. Call $H_{\{i\}}(\omega)$ the polyphase matrix after one erasure. Then (49) holds. According to (42), the average MSE with one erasure is:

$$
\mathrm{MSE}_{1}=\frac{\sigma^{2}}{2 \pi M N} \sum_{i=1}^{M} \int_{-\pi}^{\pi} \operatorname{tr}\left(\left(H_{\{i\}}^{*}(\omega) H_{\{i\}}(\omega)\right)^{-1}\right) d \omega .
$$

Call

$$
\begin{aligned}
v(\omega) & =H^{*}(\omega) H(\omega), \\
v_{i}(\omega) & =H_{i}^{*}(\omega)\left(H^{*}(\omega) H(\omega)\right)^{-1} H_{i}(\omega) .
\end{aligned}
$$

Note that $v(\omega)$ is an $N \times N$ matrix, while $v_{i}(\omega)$ is a scalar. With that, (49) can be rewritten as

$$
v_{\{i\}}(\omega)=H_{\{i\}}^{*}(\omega) H_{\{i\}}(\omega)=v(\omega)-H_{i}(\omega) H_{i}^{*}(\omega) .
$$

We now find

$$
\begin{aligned}
v_{\{i\}}(\omega)^{-1} & =v(\omega)^{-1}+ \\
& +v(\omega)^{-1} H_{i}(\omega)\left(1-v_{i}(\omega)\right)^{-1} H_{i}^{*}(\omega) v(\omega)^{-1},
\end{aligned}
$$

where we used (50) with $A=v(\omega), B=H_{i}(\omega), C=1$, and $D=H_{i}^{*}(\omega)$. Taking the trace of both sides gives

$$
\begin{aligned}
\operatorname{tr}\left(v_{\{i\}}(\omega)^{-1}\right) & =\operatorname{tr}\left(v(\omega)^{-1}\right) \\
& +\left(1-v_{i}(\omega)\right)^{-1} \operatorname{tr}\left(v(\omega)^{-1} H_{i}(\omega) H_{i}^{*}(\omega) v(\omega)^{-1}[6]\right. \\
& =\operatorname{tr}\left(v(\omega)^{-1}\right) \\
& +\left(1-v_{i}(\omega)\right)^{-1} \operatorname{tr}\left(H_{i}^{*}(\omega) v(\omega)^{-2} H_{i}(\omega)\right) \\
& =\operatorname{tr}\left(v(\omega)^{-1}\right)+\frac{H_{i}^{*}(\omega) v(\omega)^{-2} H_{i}(\omega)}{1-v_{i}(\omega)}
\end{aligned}
$$

since both $H_{i}^{*}(\omega) v(\omega)^{-2} H_{i}(\omega)$ and $1-v_{i}(\omega)$ are scalars and the trace of a product is invariant to the cyclic permutation of the factors. The average MSE becomes

$$
\begin{aligned}
\mathrm{MSE}_{1}= & \frac{\sigma^{2}}{2 \pi N} \int_{-\pi}^{\pi} \operatorname{tr}\left(v(\omega)^{-1}\right) d \omega+ \\
& \frac{\sigma^{2}}{2 \pi M N} \sum_{i=1}^{M} \int_{-\pi}^{\pi} \frac{H_{i}^{*}(\omega) v(\omega)^{-2} H_{i}(\omega)}{1-v_{i}(\omega)} d \omega .
\end{aligned}
$$

The first term of the above equation is minimized if and only if the frame is tight (since $\operatorname{tr}\left(v(\omega)^{-1}\right)=$ $\left.\operatorname{tr}\left(\left(H^{*}(\omega) H(\omega)\right)^{-1}\right)=\sum_{k=1}^{N} 1 / \lambda_{k}(\omega)\right)$. We show now that the second term is minimized as well if and only if the frame is tight. We can say that

$$
\sum_{i=1}^{M} \frac{H_{i}^{*}(\omega) v(\omega)^{-2} H_{i}(\omega)}{\left(1-v_{i}(\omega)\right)} \geq \sum_{i=1}^{M} \frac{v_{i}(\omega)^{2}}{1-v_{i}(\omega)} .
$$

Here we used Lemma A.1 from [8] which is valid for SUFs and allows us to exploit the following inequality:

$$
H_{i}^{*}(\omega) v(\omega)^{-2} H_{i}(\omega) \geq\left(H_{i}^{*}(\omega) v(\omega)^{-1} H_{i}(\omega)\right)^{2}=v_{i}(\omega)^{2} .
$$

Since we have the constraint:

$$
\sum_{i=1}^{M} v_{i}(\omega)=N
$$

the equality and minimization of (51) occur if and only if the original frame is an SUTF. This condition minimizes the maximum error too. The arguments are identical to those in [8]; we refer the reader to [8] for more details.

\section{ACKNOWLEDGMENTS}

We thank Emina Šoljanin for pointing out Naimark's Theorem. We are grateful to anonymous reviewers for their constructive comments.

\section{REFERENCES}

[1] R. M. Gray and A. D. Wyner. Source coding for a simple network. Bell System Technical Journ., 53(9):1681-1721, November 1974

[2] A. D. Wyner. On source coding with side information at the decoder. IEEE Trans. Inform. Th., IT-21(3):294-300, May 1975.

[3] A. D. Wyner. The rate-distortion function for source coding with side information at the decoder. IEEE Trans. Inform. Th., IT-22(1):1-10, January 1976

[4] J. K. Wolf, A. D. Wyner, and J. Ziv. Souce coding for multiple descriptions. Bell System Technical Journ., 59(8):1417-1426, October 1980

[5] H.S. Witsenhausen and A. D. Wyner. Souce coding for multiple descriptions II: A binary source. Bell System Technical Journ., 60(10):2281-2292, December 1981.

[6] V. K Goyal. Multiple description coding: Compression meets the network. IEEE SP Mag., 18(5):74-93, September 2001. V.K Goyal, J. Kovačević, and M. Vetterli. Multiple description transform coding: Robustness to erasures using tight frame expansions. In Proc. IEEE Int. Symp. on Inform. Th., Cambridge, MA, August 1998.

[8] V.K Goyal, J. Kovačević, and J.A. Kelner. Quantized frame expansions with erasures. Journal of Appl. and Comput. Harmonic Analysis, 10(3):203-233, May 2001.

[9] R. J. Duffin and A. C. Schaeffer. A class of nonharmonic Fourier series. Trans. Amer. Math. Soc., 72:341-366, 1952.

[10] D. Gabor. Theory of communication. Journ. IEE, 93:429-457, 1946. 
[11] I. Daubechies, A. Grossman, and Y. Meyer. Painless nonorthogonal expansions. Journ. Math. Phys., 27:1271-1283, November 1986.

[12] P.G. Casazza. The art of frame theory. Taiwanese Journ. of Math., 4(2):129-202, 2000.

[13] C. Heil and D. Walnut. Continuous and discrete wavelet transforms. SIAM Rev., 31:628-666, 1989.

[14] P.G. Casazza. Modern tools for Weyl-Heisenberg (Gabor) frame theory. Adv. in Imag. and Electron. Physics, 115:1-127, 2000.

[15] I. Daubechies. The wavelet transform, time-frequency localization and signal analysis. IEEE Trans. Inform. Th., 36(5):9611005, September 1990.

[16] I. Daubechies. Ten Lectures on Wavelets. SIAM, Philadelphia, PA, 1992.

[17] Z. Cvetković and M. Vetterli. Oversampled filter banks. Signal Proc., 46(5):1245-1255, May 1998. Submitted.

[18] H. Bolcskei, F. Hlawatsch, and H.G. Feichtinger. Frametheoretic analysis of oversampled filter banks. IEEE Trans. Signal Proc., 46(12):3256-3269, December 1998.

[19] T. Strohmer. Modern Sampling Theory: Mathematics and Applications, chapter Finite and infinite-dimensional models for oversampled filter banks, pages 297-320. Birkhauser, Boston, 2000.

[20] V. K Goyal, M. Vetterli, and N. T. Thao. Quantized overcomplete expansions in $\mathbb{R}^{N}$ : Analysis, synthesis, and algorithms. IEEE Trans. Inform. Th., 44(1):16-31, January 1998.

[21] Z. Cvetković. Resilience properties of redundant expansions under additive noise and quantization. IEEE Trans. Inform. Th. To appear.

[22] J.J. Benedetto and D. Colella. Wavelet analysis of spectogram seizure chips. In Proc. SPIE Conf. on Wavelet Appl. in Signal and Image Proc., pages 512-521, San Diego, CA, July 1995.

[23] J.J. Benedetto and G.E. Pfander. Wavelet periodicity detection algorithms. In Proc. SPIE Conf. on Wavelet Appl. in Signal and Image Proc., pages 48-55, San Diego, CA, July 1998.

[24] M. Unser. Texture classification and segmentation using wavelet frames. IEEE Trans. Image Proc., 4:1549-1560, November 1995.

[25] B. Hochwald, T. Marzetta, T. Richardson, W. Sweldens, and R. Urbanke. Systematic design of unitary space-time constellations. IEEE Trans. Inform. Th., 2000. Submitted.

[26] N. I. Akhiezer and I. M. Glazman. Theory of Linear Operators in Hilbert Spaces, volume 1. Frederick Ungar Publisher, 1966.

[27] D. Han and D.R. Larson. Frames, bases and group representations. Number 697 in Memoirs AMS. American Mathematical Society, Providence, RI, 2000.

[28] Y. Eldar and G.D. Forney, Jr. Optimal tight frames and quantum measurement. Preprint, 2001.

[29] E. Šoljanin. Frames and quantum information theory. Preprint, 2000.

[30] P.L. Dragotti, S.D. Servetto, and M. Vetterli. Optimal filter banks for multiple description coding: Analysis and synthesis. IEEE Trans. Inform. Th. To appear.

[31] S. Mehrotra. Optimal overcomplete subband expansions for multiple description quantization. In Presented at SPIE Conf. on Wavelet Appl. in Signal and Image Proc., San Diego, CA, August 2000.

[32] X. Yang and K. Ramchandran. Optimal subband filter banks for multiple description coding. IEEE Trans. Inform. Th., 46:24772490, November 2000.

[33] M. Vetterli and J. Kovačević. Wavelets and Subband Coding. Signal Processing. Prentice Hall, Englewood Cliffs, NJ, 1995.

[34] P. J. Burt and E. H. Adelson. The Laplacian pyramid as a compact image code. IEEE Trans. Commun., 31(4):532-540, April 1983.

[35] Z. Cvetković. Overcomplete Expansions for Digital Signal Processing. PhD thesis, University of California at Berekely, 1995.

[36] P. P. Vaidyanathan. Multirate Systems and Filter Banks. Prentice Hall, Englewood Cliffs, NJ, 1992.

[37] P.G. Casazza and J. Kovačević. Uniform tight frames with erasures. Advances in Computational Mathematics, special issue on Frames, 2002.

[38] P.G. Casazza and J. Kovačević. Uniform tight frames for signal processing and communications. In Proc. SPIE Conf. on Wavelet Appl. in Signal and Image Proc., pages 512-521, San Diego, CA, July 2001.

[39] S. Rangan and V. K Goyal. Recursive consistent estimation with bounded noise. IEEE Trans. Inform. Th., 47(1):457-464, January 2001.
[40] V.K Goyal. Single and Multiple Description Transform Coding with Bases and Frames. SIAM, 2002.

[41] H. Bolcskei and Y. Eldar. Geometrically uniform frames. Preprint, 2001.

[42] A. Lozano, J. Kovačević, and M. Andrews. Quantized frame expansions in a wireless environment. In Proc. Data Compr. Conf., Snowbird, Utah, March 2001. Submitted.

Jelena Kovačević (S'87-M'91-SM'96-F'02) Jelena Kovačević received the Dipl. Electr. Eng. degree from the Electrical Engineering Department, University of Belgrade, Yugoslavia, in 1986, and the Master of Science and Ph.D. degrees from Columbia University, New York, NY, in 1988 and 1991, respectively.

In November 1991, she joined AT\&T Bell Laboratories (now Lucent Technologies), Murray Hill, NJ, as a Member of Technical Staff. In the Fall of 1986, she was a Teaching Assistant at the University of Belgrade. From 1987 to 1991, she was a Graduate Research Assistant at Columbia University. In the summer of 1985, she worked for Gaz de France, Paris, France, during the summer of 1987 for INTELSAT, Washington, D.C., and in the summer of 1988 for Pacific Bell, San Ramon, CA. She is also an Adjunct Professor at Columbia University. Her research interests include wavelets, multirate signal processing, data compression and signal processing for communications.

Dr. Kovačević received the Belgrade October Prize, highest Belgrade prize for student scientific achievements awarded for the Engineering Diploma Thesis in October 1986 and the E.I. Jury Award at Columbia University for outstanding achievement as a graduate student in the areas of systems, communication or signal processing. She is a co-author (with Martin Vetterli) of the book "Wavelets and Subband Coding", Prentice Hall, 1995. She is the Fellow of the IEEE and the Editor-in-Chief of the IEEE Transactions on Image Processing. She served as an Associate Editor of the IEEE Transactions on Signal Processing, as a Guest Co-Editor (with Ingrid Daubechies) of the special issue on Wavelets of the Proceedings of the IEEE and as a Guest Co-Editor (with Martin Vetterli) of the special issue on Transform Coding of the Signal Processing Magazine. She is on the Editorial Boards of the Journal of Applied and Computational Harmonic Analysis, Journal of Fourier Analysis and Applications and the Signal Processing Magazine. Dr. Kovačević is on the IEEE Signal Processing Society Board of Governors and served on the IMDSP Technical Committee of the Signal Processing Society of the IEEE. She was a General Co-Chair (with Jan Allebach) of the Ninth Workshop on Image and Multidimensional Signal Processing.

Pier Luigi Dragotti Pier Luigi Dragotti received the Laurea Degree (summa cum laude) in Electrical Engineering in March 1997 from the University Federico II, Naples, Italy. From October 1997 to July 1998, he attended the Doctoral School in Communications Systems at the Swiss Federal Institute of Technology, Lausanne, Switzerland, where he is currently a $\mathrm{PhD}$ student in the Audio Visual Communications Laboratory. From March to September 1996 he was a visiting student at Stanford University and from July to October 2000 he was an intern at Lucent Technologies' Bell Laboratories. His research interests include joint source-channel coding, wavelets, data compression and multirate signal processing.

Vivek K Goyal (S'92-M'98) received the B.S. degree in mathematics and the B.S.E. in electrical engineering (both with highest distinction), in 1993, from the University of Iowa, Iowa City. He received the M.S. and Ph.D. degrees in electrical engineering from the University of California, Berkeley, in 1995 and 1998, respectively.

He was a Research Assistant in the Laboratoire de Communications Audiovisuelles at École Polytechnique Fédérale de Lausanne, Switzerland, in 1996. He worked in the Mathematics of Communications Research Department of Lucent Technologies' Bell Laboratories as an intern in 1997 and again as a Member of Technical Staff from 1998 to 2001. He is currently a Senior Research Engineer for Digital Fountain, Inc., Fremont, CA. His research interests include 
source coding theory, quantization theory, and practical, robust network content delivery.

Dr. Goyal is a member of Phi Beta Kappa, Tau Beta Pi, Sigma Xi, Eta Kappa Nu and SIAM. In 1998 he received the Eliahu Jury Award of the University of California, Berkeley, awarded to a graduate student or recent alumnus for outstanding achievement in systems, communications, control, or signal processing. He serves on the program committee of the IEEE Data Compression Conference. 